\title{
Polyakov Loop Susceptibility and Correlators in the Chiral Limit
}

\author{
David A. Clarke* \\ Fakultät für Physik, Universität Bielefeld, D-33615 Bielefeld, Germany \\ E-mail: dclarke@physik.uni-bielefeld.de \\ Olaf Kaczmarek \\ Fakultät für Physik, Universität Bielefeld, D-33615 Bielefeld, Germany; \\ Key Laboratory of Quark \& Lepton Physics (MOE) and Institute of Particle Physics, \\ Central China Normal University, Wuhan 430079, China \\ E-mail: okacz@physik.uni-bielefeld.de
}

\section{Frithjof Karsch}

Fakultät für Physik, Universität Bielefeld, D-33615 Bielefeld, Germany

E-mail: karsch@physik. uni-bielefeld.de

\section{Anirban Lahiri}

Fakultät für Physik, Universität Bielefeld, D-33615 Bielefeld, Germany

E-mail: alahiri@physik.uni-bielefeld.de

\begin{abstract}
In quenched QCD the Polyakov loop is an order parameter of the deconfinement transition, but with decreasing quark mass, the peak in the Polyakov loop susceptibility becomes less pronounced, and it loses its interpretation as an indicator for deconfinement. For this $N_{f}=2+1$ HISQ study, we fix the strange quark mass $m_{s}$ at its physical value and investigate the dependence of the Polyakov loop on the light quark mass $m_{l}$ in the range $m_{s} / m_{l}=27-160$, following $m_{l}$ toward the chiral limit. In particular we will look how the inflection point and susceptibility behave as we decrease $m_{l}$, to see whether one finds any indication of a crossover, and therefore whether the Polyakov loop is sensitive to the chiral phase transition. Preliminary results show no signal of a crossover from the real part of the Polyakov loop in the vicinity of the chiral crossover. Closely related is an investigation of Polyakov loop correlations and the Debye mass in this limit. Preliminary results suggest little or no dependence on $m_{l}$.
\end{abstract}

37th International Symposium on Lattice Field Theory - Lattice2019

16-22 June 2019

Wuhan, China

${ }^{*}$ Speaker. 


\section{The Polyakov loop}

For lattice QCD in a finite volume $N_{\sigma}^{3} \times N_{\tau}$, the thermal Wilson line $L_{\vec{x}}$, the Polyakov loop $P_{\vec{x}}$, and its spatial average $P$ are given by

$$
L_{\vec{x}} \equiv \prod_{\tau} U_{4}(\vec{x}, \tau), \quad P_{\vec{x}} \equiv \frac{1}{3} \operatorname{tr} L_{\vec{x}}, \quad \text { and } \quad P \equiv \frac{1}{N_{\sigma}^{3}} \sum_{\vec{x}} P_{\vec{x}},
$$

respectively. In general we define a susceptibility $\chi_{O}$ for an intensive observable $O$ as

$$
\chi_{O}=N_{\sigma}^{3}\left(\left\langle O^{2}\right\rangle-\langle O\rangle^{2}\right) .
$$

The Polyakov loop susceptibility and the susceptibility of the real part of the Polyakov loop are then

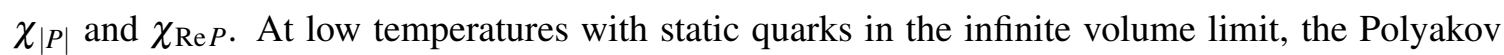
loop expectation value $\langle|P|\rangle$ is zero due to the global $\mathbb{Z}_{3}$ symmetry of the gauge action. At higher temperatures this center symmetry is spontaneously broken, and $\langle|P|\rangle$ acquires a nonzero value, signalling a finite static quark-antiquark free energy at large separations, and hence deconfinement. At finite $N_{\sigma},\langle|P|\rangle$ as a function of temperature has an inflection point, and the slope at this point diverges in the infinite volume limit. The susceptibility as a function of temperature, meanwhile, exhibits a pronounced peak at finite $N_{\sigma}$ whose height diverges in the infinite volume limit as $\chi_{|P|}^{\max } \sim$ $N_{\sigma}^{3}$, reflecting the first order nature of the deconfinement phase transition in pure $\mathrm{SU}(3)$ gauge theory.

At finite quark mass, the Polyakov loop is no longer a strict order parameter; the finite quark mass breaks the $\mathbb{Z}_{3}$ symmetry explicitly, and $\langle|P|\rangle$ and $\langle\operatorname{Re} P\rangle$ are never zero. Nevertheless at largerthan-physical quark mass, some remnants of critical behavior seem to remain, and in particular, past studies have found inflection points for $\langle|P|\rangle$ and the chiral condensate to appear at similar temperatures. For example a 2008 study [1] using improved staggered fermions of $N_{f}=2+1$ flavors, with light quark masses corresponding to about a 50\% larger-than-physical pion mass, performed on coarse lattices with $N_{\tau}=4$ and 6, found inflection points in both $\langle|P|\rangle$ and the chiral order parameter to lie around the same temperature.

While these earlier studies of Polyakov loop expectation values seemed to clearly indicate that the inflection point of the Polyakov loop is related to the chiral transition, only the latter has a clear-cut interpretation as a phase transition in the chiral limit. This "coincidence" of inflection points in the temperature dependence of $\langle|P|\rangle$ and the chiral condensate is often taken as evidence for the coincidence of a chiral and deconfinement transition. To what extent deconfinement or the melting of bound states in general can be associated with properties of the Polyakov loop at finite quark mass is, however, an open question. In fact, studies with improved fermion actions in general showed that the QCD transition, which is a pseudo-critical crossover transition at non-zero values of the quark masses, tends to become a smoother transition when going closer to the continuum limit and using more highly improved fermion actions such as HISQ or stout actions [3]. Indeed a potential hint that this behavior weakens was already given in Ref. [2], which we show in Figure 1, where ones sees that $\chi_{|P|}^{\max }$ decreases with decreasing quark mass for fixed $N_{\tau}$. Moreover there are studies with more highly improved actions that do not find the inflection point of $\langle|P|\rangle$ to coincide with the chiral inflection point $[4,5]$, challenging the notion of using the Polyakov loop as an 




Figure 1: Dependence of peak heights of Polyakov loop and chiral susceptibilities on $N_{\tau}=4$ lattices with $N_{\sigma}=8,12$, and 16 as a function of the pseudo-scalar mass in units of the vector meson mass, which is another way of stating quark mass. This calculation used a standard staggered fermion discretization scheme. Figure taken from Ref. [2].

observable for deconfinement of light degrees of freedom. In actuality, then, it is not clear to what extent properties of the Polyakov loop can provide a reasonable criterion for deconfinement.

Therefore one aim of the present study is to investigate whether an indication of deconfinement from the Polyakov loop, if any, weakens as we lower the light quark mass $m_{l}$. We employ an improved staggered action (HISQ) that leads to greatly reduced taste violations, thus providing a better approach to the continuum limit. We analyze the Polyakov loop and Polyakov loop correlation functions on lattices with temporal extent $N_{\tau}=8$ and 12, which in other thermodynamics calculations with HISQ have been shown to provide results close to the continuum limit [6].

\section{Debye screening}

The Polyakov loop relates to $F_{q \bar{q}}$, the color-averaged free energy of a static quark-antiquark pair in equilibrium at temperature $T$, by

$$
\exp \left[-F_{q \bar{q}}(r, T) / T\right]=\left\langle P_{\vec{x}} P_{\vec{y}}^{\dagger}\right\rangle \stackrel{r \rightarrow \infty}{\sim}|\langle P\rangle|^{2}=\langle\operatorname{Re} P\rangle^{2}, \quad r T=|\vec{x}-\vec{y}| / a N_{\tau},
$$

because the expectation value of the Polyakov loop lies in the real $\mathbb{Z}_{3}$ sector for finite quark masses. The gauge-invariant color-averaged Polyakov loop correlator can be decomposed into (in general gauge-dependent) color singlet and color octet contributions [7, 8, 9]. In particular the color singlet

$$
F_{1}(r, T)=-T \log \left\langle\frac{1}{3} \operatorname{tr} L_{\vec{x}} L_{\vec{y}}^{\dagger}\right\rangle,
$$

which clearly depends on the gauge. Hence it is important to restrict to a particular gauge before measuring $F_{1}$. In the deconfined phase, the interaction between two charges is screened by the medium. The distance at which in-medium modifications of the quark-antiquark interaction dominate is characterized by the Debye screening radius $r_{D}$. Its inverse, the Debye screening mass $m_{D}$, can be extracted from the long-distance $(r T \gg 1)$ behavior of $F_{1}$ as

$$
F_{1}(r, T) \simeq-\frac{4}{3} \frac{\alpha(T)}{r} e^{-r m_{D}(T)}+F_{1}(r=\infty, T) .
$$




\begin{tabular}{lcc|ccr}
\hline \hline$N_{\sigma}^{3} \times N_{\tau}$ & $m_{s} / m_{l}$ & avg. \# TU & $N_{\sigma}^{3} \times N_{\tau}$ & $m_{s} / m_{l}$ & avg. \# TU \\
\hline $32^{3} \times 8$ & 27 & 62000 & $60^{3} \times 12$ & 40 & 30000 \\
$40^{3} \times 8$ & 40 & 52000 & & 80 & 17000 \\
$56^{3} \times 8$ & 80 & 20000 & & & \\
& 160 & 17000 & & & \\
\hline \hline
\end{tabular}

Table 1: Summary of parameters used in these proceedings and corresponding statistics, reported in average molecular dynamic time units (TU) per parameter combination.

The Debye mass is known to depend on $N_{f}$ at high temperatures through a leading order perturbative calculation. At lower temperatures, lattice calculations still show qualitative agreement with perturbation theory [10]. The Debye mass will also depend on $m_{l}$; hence another goal of this study will be to see how $m_{D}$ changes with $m_{l}$.

\section{Setup and simulation parameters}

We use for our analysis configurations that were generated with the HISQ action in $(2+1)$ flavor QCD with a physical value of the strange quark mass and light quark masses in the range $m_{l}=m_{s} / 27$ to $m_{s} / 160$, corresponding to $140 \mathrm{MeV} \gtrsim m_{\pi} \gtrsim 58 \mathrm{MeV}$ [11]. The bare coupling $\beta$ is taken in the range 6.260-6.850 depending on the lattice, which was chosen so that the temperatures would lie in the vicinity of the chiral pseudo-critical temperature. Measurements of the free energies are made in the Coulomb gauge, with the gauge fixing carried out using the over-relaxation algorithm [12]. The scale has been set using the experimental value $f_{K}=156.1 / \sqrt{2} \mathrm{MeV}$ [13] as well as the updated results for the kaon decay constant calculated with the HISQ action, $f_{K} a(\beta)$, given in Ref. [14]. We renormalize the Polyakov loop using the $q q$-scheme [15], where the $T=0$ potential is obtained from a fit of the combined data shown in Fig. 14 of Ref. [6] using the ansatz

$$
V_{T=0}(r)=\frac{A}{r}+B+C r
$$

Additive renormalization constants have been calculated at $(r / a)^{2}=4$. Distances up to $(r / a)^{2}=13$ have been tree-level corrected [16]. Error bars are calculated in 32 jackknife bins unless otherwise stated. The lattice sizes, quark masses, and statistics are summarized in Table 1.

\section{Results}

The real part of the renormalized Polyakov loop is shown for different values of $m_{s} / m_{l}$ in the two plots in the top row of Figure 2 for $N_{\tau}=8$ (left) and $N_{\tau}=12$ (right) lattices. We plot this instead of $\left\langle\left|P^{\text {ren }}\right|\right\rangle$ because $\left\langle\left|P^{\text {ren }}\right|\right\rangle$ receives significant finite volume corrections from $\operatorname{Im} P$, and because after explicit breaking of the $\mathbb{Z}_{3}$ symmetry by finite quark mass, the system settles in a region where $P$ is centered on the real axis. Vertical lines indicate the positions of chiral pseudocritical temperatures taken from Ref. [11], which are at roughly 151,154 , and $158 \mathrm{MeV}$ for $N_{\tau}=8$, and 149 and $156 \mathrm{MeV}$ for $N_{\tau}=12$. In the entire temperature range probed by us $\left\langle\operatorname{Re} P^{\mathrm{ren}}\right\rangle$ is convex for both $N_{\tau}$. Calculations performed with other lattice sizes similarly show no indication that a 

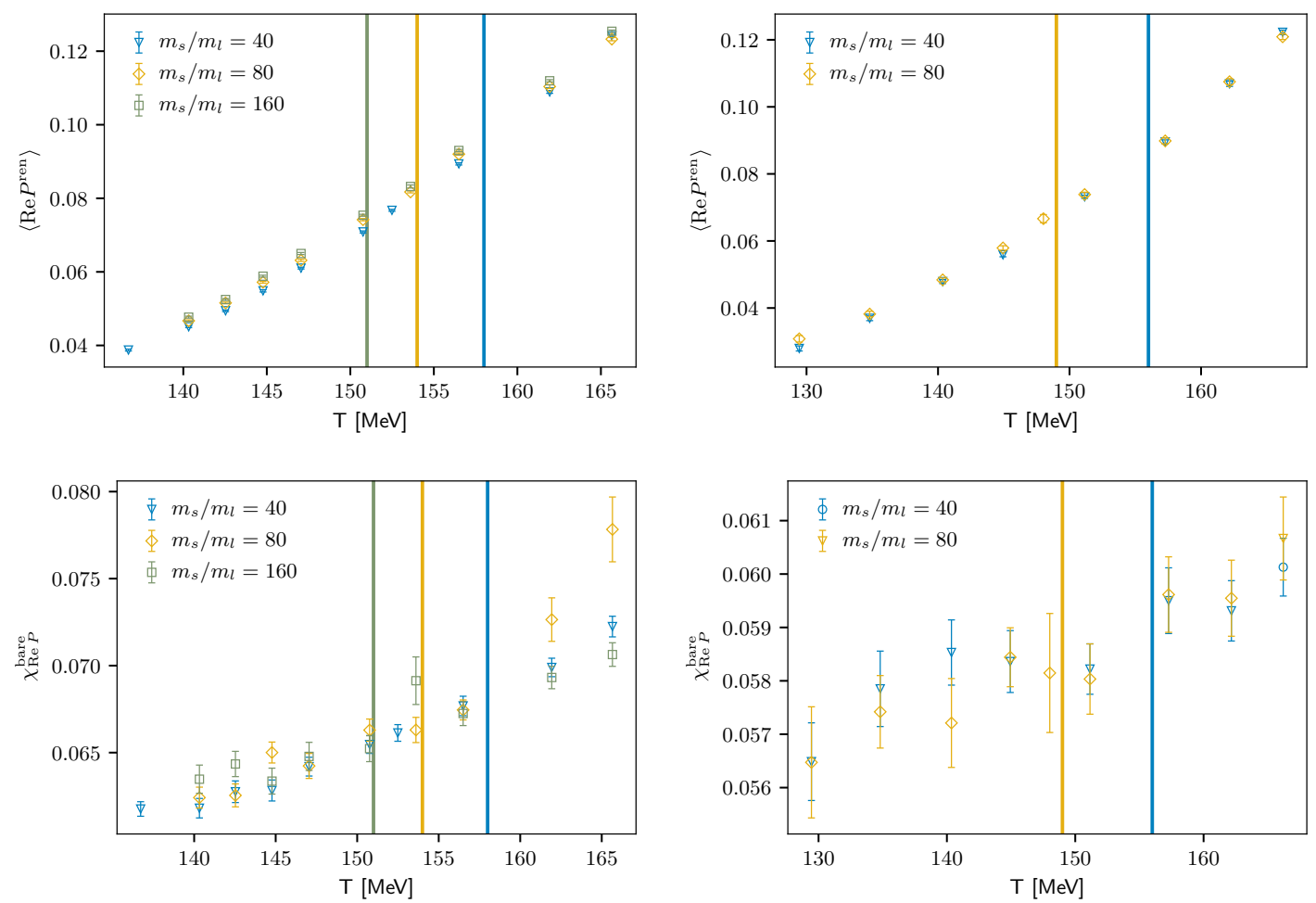

Figure 2: Real part of the renormalized Polyakov loop (top) and the susceptibility of the real part of the bare Polyakov loop (bottom) near the chiral pseudo-critical temperature for $N_{\tau}=8$ (left) and $N_{\tau}=12$ (right) for different $m_{s} / m_{l}$. The vertical lines indicate the chiral pseudo-critical temperatures corresponding to each quark mass ratio.

crossover signal from the Polyakov loop coincides with the signal from the chiral condensate. An inflection point, which needs to exist as $\left\langle\left|P^{\text {ren }}\right|\right\rangle$ (or equivalently at $N_{\sigma}=\infty,\langle\operatorname{Re} P\rangle$ ) will eventually approach unity at high temperature, will therefore only occur at temperatures larger than ${ }^{1.1} T_{p c}$. For $N_{\tau}=8$, decreasing $m_{l}$ leads to an increase in $\left\langle\operatorname{Re} P^{\text {ren }}\right\rangle$ across all probed temperatures, with no indication that the slope changes. Meanwhile for $N_{\tau}=12$ there is no $m_{l}$ dependence within the statistics.

The two plots in the bottom row of Figure 2 show $\chi_{\operatorname{Re} P}^{\text {bare }}$, the susceptibility of the real part of the bare Polyakov loop, for different $m_{l}$ for $N_{\tau}=8$ (left) and $N_{\tau}=12$ (right) for the same configurations. We see no peak in this range, which is consistent with what we find for $\left\langle\operatorname{Re} P^{\text {ren }}\right\rangle$. There is no sensitivity to $m_{l}$ for either $N_{\tau}$ in this range of temperatures. Our preliminary results thus show no overlap of a signal from the Polyakov loop with the chiral condensate. Higher statistics and more results at higher temperatures are needed to clearly see the effect of $m_{l}$ on $\chi_{\operatorname{Re} P}^{\max }$.

In Figure 3 we plot the renormalized singlet free energy against the Polyakov loop separation $r$ in physical units at $N_{\tau}=8$ for different $m_{s} / m_{l}$. The left plot shows results at $\beta=6.285$, which corresponds to a temperature of about $141 \mathrm{MeV}$, while the right plot has $\beta=6.445$, which is at about $166 \mathrm{MeV}$. The data are given at our largest available $N_{\sigma}$ for each $\beta$ and $m_{l}$ combination, which we find are large enough to suppress finite volume effects. For both plots one can see by eye that the long-distance results for all $m_{l}$ agree within statistical uncertainty. This suggests that 

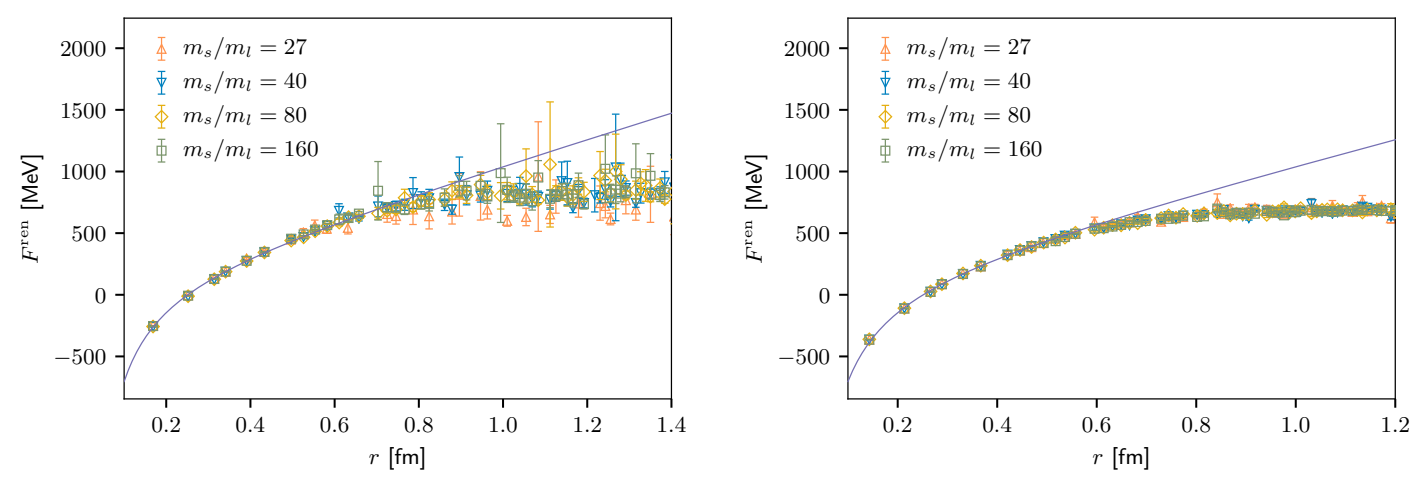

Figure 3: Dependence of $F_{1}$ on $m_{l}$ for $N_{\tau}=8$ and our largest $N_{\sigma}$ for $\beta=6.285$ or $T=141[\mathrm{MeV}]$ (left) and $\beta=6.445$ or $T=166[\mathrm{MeV}]$ (right). The purple line indicates the zero temperature potential.

the Debye screening masses, eventually extracted from these correlation functions, show little or no dependence on the light quark masses.

Extraction of $m_{D}$ has not yet yielded any precise results, as $F_{1}$ tends to be rather noisy away from short distances, which has made carrying out a long-distance fit difficult. We plan to try smoothing our configurations using the gradient flow [17], which mostly affects only short-distance physics, and could therefore improve our long-distance signal without spoiling it.

\section{Summary and Outlook}

We presented here some first results for our research on indicators of hadron melting toward the chiral limit. The Polyakov loop does not exhibit any indication of a crossover near the chiral pseudo-critical temperature at lower-than-physical quark mass, consistent with the results of more recent HISQ studies at physical $m_{l}$. More results at other $m_{l}$ are forthcoming, which will allow us to examine the behavior of $\chi_{\operatorname{Re} P}^{\max }$ and the slope of $\langle\operatorname{Re} P\rangle$ in the chiral limit. Further results at other $N_{\tau}$ will also be analyzed, allowing for a continuum limit extrapolation. We are working toward a determination of $m_{D}$, however statistical noise in the free energy at large distances make the calculation difficult. Our current data suggest $m_{D}$ will have no or little dependence on $m_{l}$ in the range investigated. Smoothing using the gradient flow may be attempted in the future.

\section{Acknowledgments}

This work was supported by the Deutsche Forschungsgemeinschaft (DFG, German Research Foundation) - project number 315477589 - TRR 211; and from the German Bundesministerium für Bildung und Forschung through Grant No. 05P18PBCA1. We thank HotQCD for providing access to their latest data sets and for many fruitful discussions. 


\section{References}

[1] M. Cheng et al., QCD equation of state with almost physical quark masses, Phys. Rev. D 77 (2008) 014511.

[2] F. Karsch, Lattice QCD at High Temperature and Density, in Lectures on Quark Matter, W. Plessas and L. Mathelitsch, eds., vol. 583, (Berlin, Heidelberg), pp. 209-249, Springer, (2002).

[3] A. Bazavov et al., Chiral and deconfinement aspects of the QCD transition, Phys. Rev. D 85 (2012) 054503.

[4] Y. Aoki et al., The QCD transition temperature: results with physical masses in the continuum limit II, J. High Energy Phys. 2009 (2009) 088.

[5] A. Bazavov et al., Polyakov loop in $2+1$ flavor QCD from low to high temperatures, Phys. Rev. D 93 (2016) 114502.

[6] A. Bazavov et al., Equation of state in (2+1)-flavor QCD, Phys. Rev. D 90 (2014) 094503.

[7] L. D. McLerran and B. Svetitsky, A Monte Carlo study of SU(2) Yang-Mills theory at finite temperature, Phys. Lett. B 98 (1981) 195.

[8] L. D. McLerran and B. Svetitsky, Quark liberation at high temperature: A Monte Carlo study of SU(2) gauge theory, Phys. Rev. D 24 (1981) 450.

[9] S. Nadkarni, Non-Abelian Debye screening. II. The singlet potential, Phys. Rev. D 34 (1986) 3904.

[10] O. Kaczmarek, Screening at finite temperature and density, PoS(CPOD07) (2008) 043.

[11] H.-T. Ding et al., Chiral Phase Transition Temperature in ( $2+1$ )-Flavor QCD, Phys. Rev. Lett. 123 (2019) 062002.

[12] J. E. Mandula and M. Ogilvie, Efficient gauge fixing via overrelaxation, Phys. Lett. B 248 (1990) 156.

[13] J. Beringer et al., Review of particle physics, Phys. Rev. D 86 (2012) 010001.

[14] A. Bazavov et al., Meson Screening Masses in (2+1)-Flavor QCD, arXiv: 1908.09552.

[15] O. Kaczmarek, F. Karsch, P. Petreczky and F. Zantow, Heavy quark-antiquark free energy and the renormalized Polyakov loop, Phys. Lett. B 543 (2002) 41.

[16] S. Necco and R. Sommer, The $N_{f}=0$ heavy quark potential from short to intermediate distances, Nucl. Phys. B 622 (2002) 328.

[17] M. Lüscher, Properties and uses of the Wilson flow in lattice QCD, J. High Energy Phys. 2010 (2010) 071. 